\title{
Vigilancia por el laboratorio de la tuberculosis resistente en menores de 15 años, Colombia, 2010 a 2015
}

\author{
Angie Zabaleta, Claudia Llerena, Angélica Valbuena \\ Grupo de Micobacterias, Dirección Redes en Salud Pública, Instituto Nacional de Salud, Bogotá, \\ D.C., Colombia
}

Introducción. La tuberculosis en los niños es un reflejo de transmisión reciente en la comunidad. Se estima que en el mundo cada año un millón de niños enferma por esta causa; en Colombia se notificaron 291 casos en el 2015.

Objetivo. Actualizar la información obtenida de las actividades de vigilancia por el laboratorio de la farmacorresistencia del bacilo Mycobacterium tuberculosis en menores de 15 años en Colombia entre el 2010 y el 2015.

Materiales y métodos. Se llevó a cabo un estudio retrospectivo de corte transversal. Se estudiaron las variables de procedencia, sexo, edad, tipo de tuberculosis y estado de HIV en los casos sensibles y resistentes. Estos se clasificaron como caso nuevo sin tratamiento o caso previamente tratado para analizar el perfil de resistencia a fármacos de primera y segunda línea.

Resultados. De los 3.440 casos notificados, en el $16,4 \%$ se practicó la prueba de sensibilidad. El 50,6 \% eran mujeres, la forma pulmonar se presentó en el 70,6 \% y el 1,4 \% presentó coinfección de tuberculosis y HIV. Se estudiaron 565 casos, de los cuales 503 (89,0 \%) eran nuevos: el 3,9\% con tuberculosis multirresistente y el $9,5 \%$ con resistencia global. Los previamente tratados fueron 62 (10,9\%), 4,8 \% con multirresistencia y 19,3\% con resistencia global. No se evidenciaron diferencias estadísticamente significativas en los años estudiados. La proporción de tuberculosis extremadamente resistente en los casos nuevos evaluados fue de $9,0 \%$.

Conclusiones. Es necesario que el Ministerio de Salud y Protección Social y el Instituto Nacional de Salud promuevan el uso de pruebas diagnósticas rápidas y muy sensibles, como las moleculares recomendadas por la Organización Mundial de la Salud.

Recibido: 25/01/18

Aceptado: 20/09/18

Publicado: $26 / 09 / 18$

Citación:

Zabaleta AP, Llerena C, Valbuena A. Vigilancia por el laboratorio de la tuberculosis resistente en menores de 15 años, Colombia, 2010 a 2015. Biomédica. 2019;39:330-8

https://doi.org/10.7705/biomedica.v39i3.4258

\section{Correspondencia:}

Angie Paola Zabaleta, Grupo de Micobacterias, Dirección Redes en Salud Pública, Instituto Nacional de Salud, Avenida calle $26 N^{\circ}$ 51-20, Bogotá, D.C., Colombia

Teléfono: (1) 220 7700, extensión 1260

azabaleta@ins.gov.co

Contribución de los autores:

Todas las autoras participaron en el diseño del protocolo, la recolección y el análisis de la información, la redacción y la revisión del artículo.

Financiación:

Este trabajo se llevó a cabo con recursos del contrato 757/13 celebrado entre el Departamento Administrativo de Ciencia y Tecnología de Colciencias y la Dirección de Redes en Salud Pública del Instituto Nacional de Salud.

Conflicto de intereses:

Las autoras del presente artículo no tienen ningún tipo de conflicto de intereses financieros, académicos ni personales, que puedan afectar su validez.
Palabras clave: Mycobacterium tuberculosis; antituberculosos; tuberculosis resistente a múltiples medicamentos; tuberculosis extensivamente resistente a drogas; niño; Colombia.

\section{Resistant tuberculosis in children under 15 years of age, Colombia 2010-2015}

Introduction: Tuberculosis in children is a recent transmission reflection in the community. It is estimated that every year one million children get sick in the world because of this. In Colombia, 291 cases were notified in 2015.

Objective: To update the information obtained from the surveillance activities of the drugresistance laboratory in children younger than 15 years of age in Colombia between 2010 and 2015.

Materials and methods: This was a cross-sectional retrospective study. We studied the variables of origin, gender, age, type of tuberculosis, and HIV status in sensitive and resistant cases. We classified them according to their treatment background between new and previously treated to analyze their first and second line drug resistance profile.

Results: From the notified cases, $16.4 \%$ had a sensitivity test. $50.6 \%$ were women, the pulmonary form was present in $70.6 \%$ cases, and $1.4 \%$ presented with tuberculosis/HIV coinfection. We studied 565 cases, from which 503 (89.1\%) were new, presenting with multidrug-resistant tuberculosis, and a global resistance of $3.9 \%$ and $9.5 \%$, respectively. From them, 62 had been previously treated (10.9\%), with $4.8 \%$ and $19.3 \%$ multidrug resistance and global resistance, respectively. There was no evidence of statistically significant differences during the studied years. Extremely resistant tuberculosis in new cases was $9.0 \%$.

Conclusions: It is necessary for the Ministerio de Salud y Protección Social and the Instituto Nacional de Salud to promote the use of faster and more sensitive diagnostic tests such as the molecular ones recommended by the World Health Organization.

Keywords: Mycobacterium tuberculosis; antitubercular agents; tuberculosis, multidrugresistant; extensively drug-resistant tuberculosis; child; Colombia. 
Los casos de tuberculosis resistente en la población infantil reflejan la transmisión reciente de cepas de adultos en la comunidad. Se estima que cada año un millón de niños enferma por el bacilo de la tuberculosis, solo uno de cada tres es diagnosticado, el $99 \%$ de los que inician tratamiento sobrevive y el $22 \%$ de los no tratados muere (1).

En el 2015 la Organización Mundial de la Salud (OMS) notificó 6,1 millones de casos nuevos y recaídas de tuberculosis en el mundo, de los cuales el 6,3\% correspondió a menores de 15 años. Además, se registraron 132.120 casos de tuberculosis multirresistente (Multidrug-resistant TB, MDR-TB) y resistente a rifampicina, de los cuales se estima que 32.000 eran niños $(1,2)$. En ese mismo año en Colombia se notificaron 12.918 casos de todas las formas de tuberculosis al Sistema de Vigilancia en Salud Pública (Sivigila); de estos, 291 (2,2\%) correspondieron a menores de 15 años y tres presentaron tuberculosis multirresistente (3).

En el 2010, Llerena, et al., describieron la situación de la tuberculosis resistente en los menores de 15 años en Colombia; la resistencia global en los niños sin antecedentes de tratamiento fue de $21,1 \%$ y en aquellos con tuberculosis multirresistente, de 6,5\%; no se encontró resistencia en los previamente tratados (4).

En Colombia son pocos los estudios realizados para conocer la prevalencia de la resistencia a los fármacos en la población infantil. El objetivo del presente trabajo fue actualizar la información obtenida de las actividades de vigilancia por el laboratorio de la farmacorresistencia en personas menores de 15 años en Colombia en un periodo de seis años.

\section{Materiales y métodos}

Se llevó a cabo un estudio retrospectivo de corte transversal en menores de 15 años con tuberculosis pulmonar o extrapulmonar entre el 2010 y el 2015 en Colombia.

Se incluyeron 565 menores de 15 años que tenían un cultivo positivo para el complejo Mycobacterium tuberculosis y una prueba de sensibilidad a los fármacos antituberculosis de primera y segunda línea con resultado de sensible o resistente; 35 no pudieron interpretarse o se habían contaminado.

Las fuentes de información fueron el formato único de vigilancia de las micobacterias, que se recibe en el Laboratorio Nacional de Referencia con los datos básicos de cada persona, la base de datos de pruebas de sensibilidad del mismo laboratorio y los informes de tuberculosis del Sivigila.

Para el análisis de los datos, los casos se dividieron en tres grupos: con tuberculosis sensible a la isoniacida y la rifampicina, con tuberculosis resistente a la isoniacida, o con tuberculosis multirresistente, resistente a la rifampicina o ambos. Se analizaron las siguientes variables: procedencia del cultivo según la entidad territorial, sexo, edad, tipo de tuberculosis, muestra procesada, coinfección con HIV y patrón de sensibilidad a los fármacos de primera y segunda línea.

Se calcularon las medidas de tendencia central y dispersión para las variables numéricas y porcentajes para las categóricas, diferenciando entre los tres grupos. Se practicaron las pruebas de diferencia de proporciones y de medias para determinar las diferencias de las variables entre estos grupos, con una confianza del $95 \%$ y una significación estadística de $p<0,05$. 
Para el análisis del perfil de resistencia a los fármacos antituberculosis, se diferenció entre casos nuevos y previamente tratados, se calcularon los porcentajes de los casos con prueba de sensibilidad a los fármacos de primera línea con respecto a lo notificado por el Sivigila, los casos de tuberculosis multirresistente y resistente a la rifampicina con pruebas de sensibilidad a los fármacos de segunda línea, resistencia global a los fármacos de primera (isoniacida y rifampicina) y segunda línea (kanamicina, amikacina, capreomicina y ofloxacina), la resistencia por cada fármaco, la tuberculosis multirresistente y resistente a la rifampicina y la tuberculosis extremadamente resistente (Extensively Drug-Resistant Tuberculosis, XDRTB) entre los casos multirresistentes.

Las metodologías utilizadas por la Red Nacional de Laboratorios para determinar la sensibilidad a los fármacos antituberculosis fueron: proporciones múltiples en medio de Löwenstein-Jensen y en capa delgada, Bactec MGIT'M , Genotype MTBDR plus V2 ${ }^{\mathrm{TM}}$ y Xpert MTB RIFTM.

Los datos se organizaron, se tabularon y se analizaron con las herramientas de Excel ${ }^{\mathrm{TM}}$ y Epidat 3.1 ${ }^{\mathrm{TM}}$.

\section{Aspectos éticos}

Según la Resolución 008430 de 1993 del Ministerio de Salud y Protección Social, la información obtenida por el Instituto Nacional de Salud en este estudio hace parte de las actividades de vigilancia de la farmacorresistencia, y el estudio se clasifica como una investigación sin riesgo debido a que es un análisis retrospectivo y no se hizo ninguna intervención intencionada de las variables biológicas, fisiológicas, sicológicas o sociales de los menores.

\section{Resultados}

Al comparar los casos notificados al Sivigila de los menores de 15 años y las pruebas de sensibilidad a los fármacos antituberculosis, se encontró que se conocía el perfil de resistencia solo en $82(13,1 \%)$ de los 624 casos notificados en el 2010, en $83(11,6 \%)$ de los 714 casos en el 2011, en $96(15,1 \%)$ de los 636 casos en el 2012, en 104 (18,1\%) de los 575 casos en el 2013, en 110 (18,3\%) de los 600 casos en el 2014 y en 90 (30,9\%) de los 291 en el 2015.

En total, durante los seis años analizados se notificaron 3.440 casos al Sivigila, a $565(16,4 \%)$ de los cuales se les practicaron pruebas de sensibilidad a los fármacos. Se observó un incremento a partir del 2013 con respecto a lo registrado en los dos años anteriores $(p<0,05)$ y el mayor porcentaje de evaluados se alcanzó en el $2015(p<0,05)(3,5-9)$.

De estos 565 casos, 286 (50,6 \%) correspondía a mujeres y no se encontraron diferencias estadísticas entre los tres grupos etarios evaluados (cuadro 1).

De los menores de 15 años analizados, el menor número de casos de tuberculosis resistente a la isoniacida se presentó en el grupo de los 6 a los 10 años $(p<0,05)$, comparado con el grupo etario de 0 a 5 , y el de 11 a 15 años. En los casos de MDR-TB o de resistencia a la rifampicina, no hubo diferencias significativas entre los tres grupos de edad $(p=0,081)$ (cuadro 1).

La tuberculosis fue pulmonar en 399 (70,6 \%) casos (cuadro 1). Estos se distribuyeron así: en el grupo de 0 a 5 años, 170 de 219 (77,6\%); en el de 6 a 10 años, 81 de 129 (63\%) y, en el de 11 a 15 años, 172 de 217 (79,2\%). La tuberculosis pulmonar fue menos frecuente en el grupo de 6 a10 años $(p<0,05)$. 
Cuadro 1. Descripción de variables sociodemográficas en 565 casos de tuberculosis en menores de 15 años, Colombia, 2010 a 2015

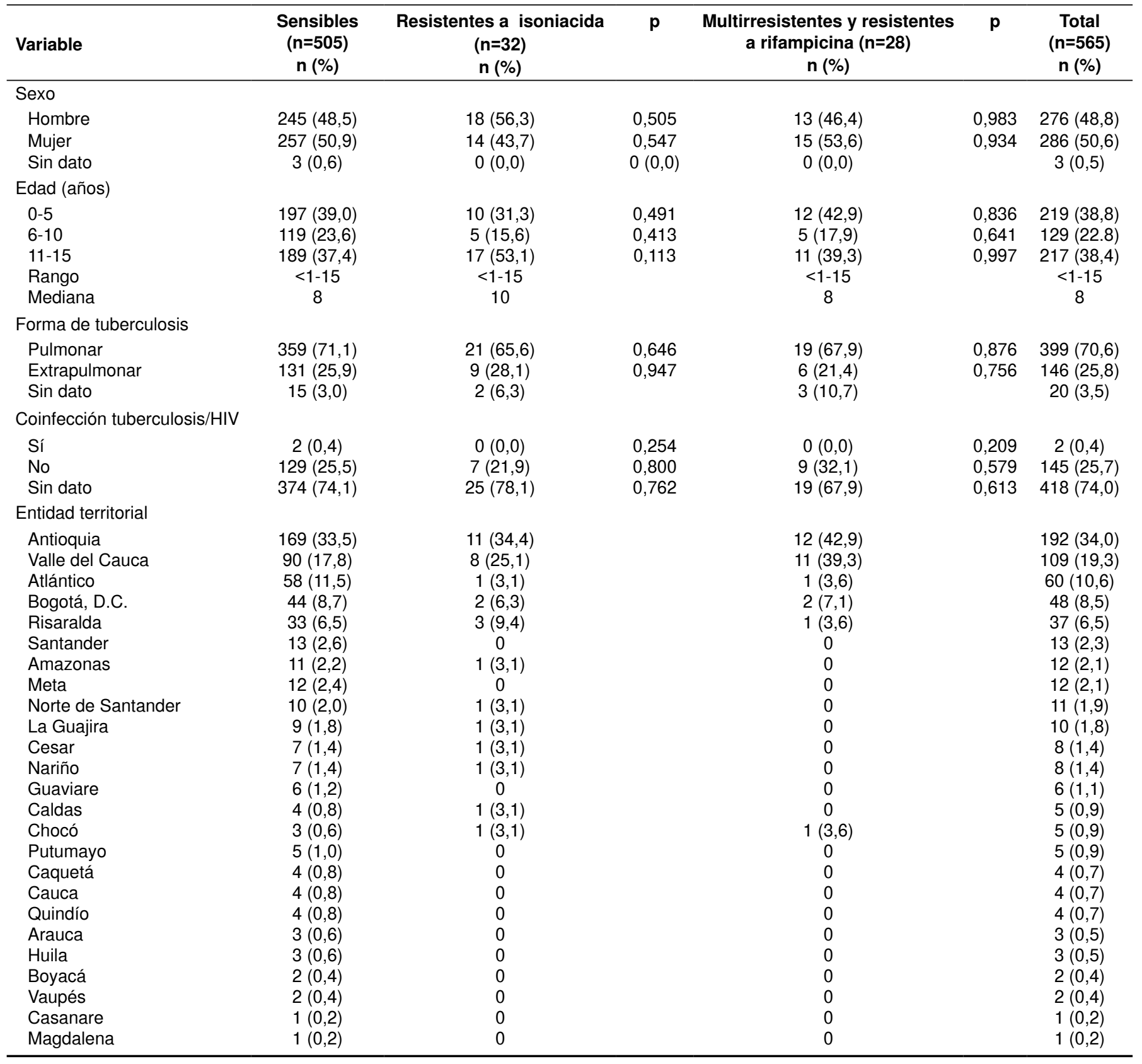

De los 146 casos de tuberculosis extrapulmonar, en $89(61 \%)$ no se informó el origen de la muestra. En 26 (17,8\%), la muestra provenía de la pleura, en $15(10,3 \%)$, de ganglios, en 7 (4,8\%), de órganos abdominales, en $8(5,5 \%)$, de la tráquea, y en $1(0,7 \%)$, del peritoneo.

El registro del resultado de la prueba de HIV solo se obtuvo en 147 (26,0 $\%$ ) casos, de los cuales 2 (1,4\%) fueron positivos y 418 (73,9\%) no tenían esta información (cuadro 1). Los dos casos positivos eran formas pulmonares de pacientes con edades entre los 11 y 14 años.

En la distribución por entidad territorial de procedencia, se encontró que el mayor aporte provenía de las áreas del país que presentan más casos de 
tuberculosis y farmacorresistencia; estas son Antioquia con 192 (34,0\%), Valle del Cauca con 109 (19,3\%) y Atlántico con 60 (10,6 \%) (cuadro 1).

De los 565 casos analizados, 503 (89,0 \%) eran nuevos. En este grupo, $455(90,4 \%)$ fueron sensibles a la isoniacida y la rifampicina $\left(\mathrm{IC}_{95 \%} 87,8-\right.$ $93,1), 26(5,2 \%)$ presentaron resistencia a la isoniacida $\left(\mathrm{IC}_{95 \%} 3,1-7,2\right), 2$ $(0,4 \%)$ a la rifampicina $\left(\mathrm{IC}_{95 \%} 0,05-1,4\right)$, y $20(3,9 \%)$ fueron multirresistentes $\left(\mathrm{IC}_{95 \%} 2,2-5,8\right)$. En total, se identificaron 48 casos de resistencia, lo que corresponde a una resistencia global de 9,5\% ( $\left.\mathrm{IC}_{95 \%} 6,9-12,2\right)$, sin diferencias significativas entre los años estudiados (cuadro 2).

Cuadro 2. Vigilancia de la resistencia en casos nuevos y previamente tratados de tuberculosis en menores de 15 años, Colombia, 2010 a 2015

\begin{tabular}{|c|c|c|c|c|c|c|c|c|c|c|c|}
\hline \multicolumn{12}{|c|}{ Casos nuevos } \\
\hline \multirow{2}{*}{ Año } & \multirow{2}{*}{ Casos } & \multicolumn{10}{|c|}{ Porcentaje de resistencia } \\
\hline & & Sensible & $\mathbf{p}$ & $\mathbf{H}$ & $\mathbf{p}$ & $\mathbf{R}$ & $\mathbf{p}$ & MDR- TB & $\mathbf{p}$ & RG & $\mathbf{p}$ \\
\hline 2010 & 72 & $62(86,1 \%)$ & REF & $7(9,7 \%)$ & REF & 0 & & $3(4,2 \%)$ & REF & $10(13,9 \%)$ & REF \\
\hline 2011 & 61 & $55(90,2 \%)$ & 0,653 & $1(1,6 \%)$ & 0,112 & 0 & & $5(8,2 \%)$ & 0,543 & $6(9,8 \%)$ & 0,653 \\
\hline 2012 & 90 & $82(91,1 \%)$ & 0,450 & $4(4,4 \%)$ & 0,311 & 0 & & $4(4,4 \%)$ & 0,762 & $8(8,9 \%)$ & 0,450 \\
\hline 2013 & 90 & $83(92,2 \%)$ & 0,315 & $3(3,3 \%)$ & 0,176 & $1(1,1 \%)$ & REF & $3(3,3 \%)$ & 0,889 & $7(7,8 \%)$ & 0,315 \\
\hline 2014 & 105 & $98(93,3 \%)$ & 0,179 & $4(3,8 \%)$ & 0,199 & 0 & & $3(2,8 \%)$ & 0,969 & $7(6,7 \%)$ & 0,179 \\
\hline 2015 & 85 & $75(88,2 \%)$ & 0,874 & $7(8,2 \%)$ & 0,964 & $1(1,2 \%)$ & 0,502 & $2(2,4 \%)$ & 0,850 & $10(11,8 \%)$ & 0,874 \\
\hline Total & 503 & $\begin{array}{c}455(90,4 \%) \\
87,8-93,1\end{array}$ & 0,349 & $\begin{array}{c}26(5,2 \%) \\
3,1-7,2\end{array}$ & 0,199 & $\begin{array}{c}2(0,4 \%) \\
0,05-1,4\end{array}$ & 0,942 & $\begin{array}{c}20(3,9 \%) \\
2,2-5,8\end{array}$ & 0,807 & $\begin{array}{c}48(9,5 \%) \\
6,9-2,2\end{array}$ & 0,349 \\
\hline \multicolumn{12}{|c|}{ Casos previamente tratados } \\
\hline 2010 & 10 & $9(90 \%)$ & REF & $1(10 \%)$ & REF & 0 & & 0 & & $2(20 \%)$ & REF \\
\hline 2011 & 22 & $19(86,4 \%)$ & 0,773 & $2(9,1 \%)$ & 0,567 & 0 & & $1(4,5 \%)$ & REF & $4(18,2 \%)$ & 0,714 \\
\hline 2012 & 6 & $5(88,3 \%)$ & 0,696 & $1(16,7 \%)$ & 0,696 & 0 & & 0 & & $2(33,3 \%)$ & 1,000 \\
\hline 2013 & 14 & $10(71,4 \%)$ & 0,552 & $2(14,3 \%)$ & 0,754 & $1(7,1 \%)$ & REF & $1(7,1 \%)$ & 0,678 & $4(28,6 \%)$ & 1,000 \\
\hline 2014 & 5 & 4 (80 \%) & 0,788 & 0 & & $1(20 \%)$ & 0,964 & 0 & & $1(20 \%)$ & 0,493 \\
\hline 2015 & 5 & $3(60 \%)$ & 0,493 & 0 & & $1(20 \%)$ & 0,964 & $1(20 \%)$ & 0,806 & $2(40 \%)$ & 0,836 \\
\hline Total & 62 & $\begin{array}{c}50(80,6 \%) \\
70-91,3\end{array}$ & 0,786 & $\begin{array}{c}6(9,7 \%) \\
1,5-17,8\end{array}$ & 0,587 & $\begin{array}{c}3(4,8 \%) \\
1,0-13,5\end{array}$ & 0,753 & $\begin{array}{c}3(4,8 \%) \\
1,0-13,5\end{array}$ & 0,598 & $\begin{array}{c}12(19,3 \%) \\
8,7-29,9\end{array}$ & 0,702 \\
\hline
\end{tabular}

H: isoniacida; R: rifampicina; MDR-TB: tuberculosis multirresistente ; RG: resistencia global; REF: grupo de comparación

Con antecedentes de tratamiento antituberculoso se encontraron 62 $(10,9 \%)$ casos. En este grupo, $50(80,6 \%)$ fueron sensibles a la isoniacida y a la rifampicina $\left(\mathrm{IC}_{95 \%}\right.$ 70-91,3); $6(9,7 \%)$ presentaron resistencia a la isoniacida $\left(\mathrm{IC}_{95 \%}, 1,5-17,8\right)$ y $3(4,8 \%)$ a la rifampicina $\left(\mathrm{IC}_{95 \%} 1,0-13,5\right)$, y hubo multirresistencia en $3(4,8 \%)\left(\mathrm{IC}_{95 \%}, 1,0-13,5\right)$. En total, se identificaron 12 casos de resistencia, lo que corresponde a una resistencia global de $19,3 \%\left(\mathrm{IC}_{95 \%} 8,7\right.$ 29,9), sin diferencias significativas entre los años estudiados (cuadro 2).

De los 28 casos identificados con MDR-TB o resistencia a la rifampicina, $13(46,4 \%)$ tenían resultado de prueba de sensibilidad a los fármacos antituberculosis de segunda línea. Once casos eran nuevos (39,3\%), de los cuales $9(81,8 \%)$ fueron sensibles a la kanamicina, la amikacina, la capreomicina y la ofloxacina, y $3(18,2 \%)$ presentaron resistencia: un niño de dos años de edad a la ofloxacina en Antioquia y otro de 5 años con XDR-TB a la kanamicina, la amikacina y la ofloxacina en el Valle del Cauca. Es decir, de los casos nuevos, uno $(9,0 \%)$ presentó resistencia a la kanamicina y la amikacina, y dos $(18,2 \%)$ a la ofloxacina, y en uno $(9,0 \%)$ la tuberculosis era extremadamente resistente XDR-TB. Los dos casos (100\%) previamente tratados fueron sensibles a los cuatro fármacos de segunda línea evaluados. 


\section{Discusión}

El diagnóstico de tuberculosis y farmacorresistencia en la población infantil es un reto para los sistemas de salud debido a las limitaciones de los métodos diagnósticos por el laboratorio y a la inespecificidad de los síntomas en los niños, lo que favorece el subregistro y hace que no sea clara la magnitud de la enfermedad en ellos.

Algunos estudios indican que la confirmación bacteriológica se logra entre el $20 \%$ y el $50 \%$ de los que ingresan al programa de tuberculosis para tratamiento. Esto puede variar aún más si se tiene en cuenta la sensibilidad de las técnicas diagnósticas empleadas. En Colombia, generalmente son la baciloscopia y el cultivo, métodos con los cuales es difícil detectar la micobacteria debido a la escasa carga bacilar que tienen; además, la obtención de las muestras no es fácil y, en algunos casos, hay fallas en el proceso de recolección y procesamiento que pueden causar falsos negativos $(1,10-13)$.

Otras posibles causas pueden ser las barreras que existen para acceder a las diferentes metodologías, sean convencionales, moleculares o mediante cultivo en medio líquido, las dos últimas con mayor sensibilidad; estos métodos diagnósticos fueron incluidos por el Ministerio de Salud en el plan obligatorio de salud y están disponibles en el país $(14,15)$.

En el 2010, Llerena, et al., reportaron que el porcentaje de casos que contaron con un cultivo de $M$. tuberculosis y el resultado de la prueba de sensibilidad a los fármacos antituberculosis fue de 3,4\% en el 2007 y de $5,3 \%$ en el 2008. Durante el periodo de estudio del presente trabajo, este porcentaje aumentó al 11,8\% en el 2010, se mantuvo estable hasta el 2014 y aumentó a 30,9 \% en el 2015; esto se debe a que para este último año la notificación al Sivigila fue menor (3-9).

No hubo diferencias entre los sexos en lo referente a los casos de tuberculosis pediátrica, tal como se observa en los adultos y similar a lo descrito en otros estudios, en los cuales es claro que lo que sucede en los niños es un reflejo de lo que ocurre en los mayores de edad $(4,10,16,17)$.

Los grupos etarios en los que predominó la tuberculosis infantil fueron de los de 0 a 5 y de 11 a 14 años, semejante a lo publicado por Llerena, et al., quienes observaron que el $62,4 \%$ de los casos pertenecía a los mismos rangos de edad. Otros estudios consultados reportan algunas variaciones en los rangos, sin embargo, todos concuerdan en que existe un mayor riesgo de enfermar en las edades muy tempranas (menores de cinco), el cual disminuye en los niños entre los 6 y los 10 años, y aumenta de nuevo en los mayores de 11 años $(4,11,16-20)$.

La presentación clínica más común es la tuberculosis pulmonar, aunque las formas miliares y extrapulmonares son más comunes que en los adultos $y$, por ello, siempre se debe mantener la sospecha diagnóstica. En el presente trabajo, el 70,6 \% de los casos era de tuberculosis pulmonar, similar a lo reportado por otros autores $(4,10,16,20-22)$.

En Colombia, el mayor porcentaje de los casos de tuberculosis extrapulmonar corresponde a la forma pleural, seguida por la meníngea y la ganglionar. En este estudio, no se encontraron casos de tuberculosis meníngea; predominaron la pleural y la ganglionar, semejante a lo reportado en el país. Es de resaltar el alto porcentaje de casos sin información sobre el origen de la muestra (3). 
En este análisis, el porcentaje de coinfección con HIV fue de 1,4 \% entre los casos en que se registró el resultado de la prueba. No se reportó esta condición en 74,0 \% de los casos, semejante a lo encontrado en el estudio de Morcillo, et al., en el cual se presentó en el 1,6\% de los casos y no se conocía el dato en más del $70 \%$. Otros trabajos en Colombia indican una coinfección de alrededor del $6 \%$; sin embargo, esto puede variar según la incidencia de estas dos infecciones. Es evidente que existe un importante subregistro debido a la falta de información. La presencia conjunta de estas enfermedades aumenta el riesgo de diseminación, y las tasas de morbilidad y mortalidad $(4,10,11,20,22)$.

En el año 2010, Llerena, et al., reportaron que el 78,9 \% de los casos nuevos eran sensibles a los fármacos antituberculosos, la resistencia global fue de $21,1 \%$ y la MDR-TB de 6,5\%; en el presente trabajo, se evidenciaron menores porcentajes, de $9,5 \%$ y $3,9 \%$, respectivamente. Esto se puede deber a que solo el $16,4 \%$ de los casos notificados tenía pruebas de sensibilidad en el período estudiado, lo que indica un subregistro. Solo se estudiaron cinco casos previamente tratados, de los cuales uno $(20 \%)$ era de MDR-TB; en el presente análisis, la proporción fue de 4,8\%. Las diferencias se podrían deberse al número de casos evaluados con tratamiento previo en cada uno de los estudios.

En teoría, la presencia de resistencia en los menores de 15 años es el reflejo de la transmisión por un caso índice. Sin embargo, cuando ha habido una mala administración del tratamiento antituberculoso, aumenta la probabilidad de que se desarrolle una tuberculosis resistente $(4,21)$.

Es de resaltar que los datos obtenidos no reflejan un aumento de la resistencia comparado con lo reportado previamente en el país $(4,23)$.

Morcillo, et al., encontraron MDR-TB en 5,1 \% de los casos nuevos y en $3,7 \%$ de los previamente tratados, y ninguno de XDR-TB. En lo referente a la multirresistencia, los datos son similares a los aquí presentados. Sin embargo, en el presente trabajo se documenta la presencia de resistencia a los fármacos de segunda línea y el primer caso de XDR-TB en un menor de 15 años, el cual provenía del departamento del Valle del Cauca y falleció por esta causa $(4,20)$.

Ante un caso de tuberculosis en un menor de edad, es fundamental garantizar la eficacia de los medicamentos administrados. La resistencia a la isoniacida, además de convertirse en un riesgo de falla terapéutica, hace necesario saber si el paciente recibió profilaxis contra la tuberculosis en algún momento de su vida. En estas circunstancias, es fundamental diferenciar entre la enfermedad latente y la activa. En una revisión sistemática de 2015 sobre la resistencia a la isoniacida, se estimó que el 12,1\% $\left(\mathrm{IC}_{95 \%} 9,8-14,8\right)$ de los niños con tuberculosis presentaría resistencia a este medicamento; algo que también se evidenció en el presente estudio en los casos nuevos y los previamente tratados (24).

Como limitaciones del presente estudio están los criterios de exclusión de los casos. Además, se presentó un alto porcentaje de registros sin información de la prueba de HIV en la base de datos utilizada, y no se pudo establecer si los casos previamente tratados correspondían a fracaso terapéutico, pérdida del seguimiento o recaída, ni los riesgos ni las características clínicas, bacteriológicas o epidemiológicas antes del diagnóstico, durante el tratamiento y en su condición final. Todo esto impidió hacer un estudio retrospectivo de cohorte. 
Dada la relevancia de la información proporcionada por este estudio, que refleja la gran transmisibilidad de la enfermedad y las fallas de los programas de tuberculosis en todos los niveles de atención, es necesario practicar en estos casos pruebas más sensibles, como las moleculares, que permiten hacer un diagnóstico rápido y oportuno para prevenir formas graves de la enfermedad. Además, se debe mejorar la calidad del estudio de los contactos para detectar las fuentes de contagio y los casos nuevos, y fortalecer la vigilancia de la resistencia en los niños.

El Ministerio de Salud y Protección Social debe normar el uso de estas metodologías, para que a través de las empresas aseguradoras de planes de beneficios se garantice su uso, e incluirlas dentro de las rutas integrales de atención en salud $(4,15,16)$.

\section{Agradecimientos}

A la Red Nacional de Laboratorios de Tuberculosis del país, por las actividades de vigilancia de la resistencia realizadas.

\section{Referencias}

1. Jenkins H, Tolman A, Yuen C, Parr J, Keshavjee S, Pérez C, et al. Incidence of multidrugresistant tuberculosis disease in children: Systematic review and global estimates. Lancet Infect Dis. 2014;383:1572-9. https://doi.org/10.1016/S0140-6736(14)60195-1

2. World Health Organization. Global tuberculosis report, 2016. Fecha de consulta: 4 de enero del 2017. Disponible en: http://apps.who.int/iris/bitstream/10665/191102/1/9789241565059 eng.pdf

3. Instituto Nacional de Salud. Informe final del evento tuberculosis, Colombia, 2015. Fecha de consulta: 25 de febrero del 2016. Disponible en: http://www.ins.gov.co/buscador-eventos/ Informesdeevento/Tuberculosis\%202015.pdf

4. Llerena C, Fadul SE, Garzón MC, Mejía G. Resistencia de Mycobacterium tuberculosis a los fármacos antituberculosos en menores de 15 años en Colombia. Biomédica. 2010;30:36270. https://doi.org/10.7705/biomedica.v30i3.270.

5. Instituto Nacional de Salud. Comportamiento de la notificación de tuberculosis Colombia, semana 1-52 de 2010, período 13. Fecha de consulta: 25 de febrero de 2016. Disponible en: http://www.ins.gov.co/lineas-de-accion/Subdireccion-Vigilancia/Informe de Evento Epidemiolgico/Tuberculosis 2010.pdf

6. Instituto Nacional de Salud. Comportamiento de la notificación de tuberculosis Colombia, semana 1-52 de 2011, período 13, cierre. Fecha de consulta: 25 de febrero de 2016. Disponible en: http://www.ins.gov.co/lineas-de-accion/Subdireccion-Vigilancia/Informe de Evento Epidemiolgico/Tuberculosis 2011.pdf

7. Instituto Nacional de Salud. Informe del evento tuberculosis, hasta el periodo epidemiológico 13 del año 2012, cierre. Fecha de consulta: 25 de febrero de 2016. Disponible en: http://www.ins.gov.co/lineas-de-accion/Subdireccion-Vigilancia/Informe de Evento Epidemiolgico/TUBERCULOSIS 2012.pdf.

8. Instituto Nacional de Salud. Informe del evento tuberculosis, año 2013. Fecha de consulta: 25 de febrero de 2016. Disponible en: http://www.ins.gov.co/lineas-de-accion/SubdireccionVigilancia/Informe de Evento Epidemiolgico/TUBERCULOSIS 2013.pdf

9. Instituto Nacional de Salud. Informe final tuberculosis, Colombia, 2014. Fecha de consulta: 25 de febrero de 2016. Disponible en: http://www.ins.gov.co/lineas-de-accion/SubdireccionVigilancia/Informe de Evento Epidemiolgico/Tuberculosis 2014.pdf

10. Rodríguez E, Garrido M, Villarrubia S, Martpin L, Hernández G. Epidemiology of childhood tuberculosis in Spain: 2005-2009. Rev Esp Salud Pública. 2012;86:49-59.

11. Bisero E, Luque G, Borda M, Melillo K, Zapata A, Varela S. Tuberculosis en una población pediátrica atendida en un hospital público. Adherencia (sic) al tratamiento: Estudio descriptivo. Rev Amer Med Respiratoria. 2013;4:184-9.

12. Organización Panamericana de la Salud. Manual para el diagnóstico de tuberculosis. Parte 1. Baciloscopia. Fecha de consulta: 18 de junio de 2016. Disponible en: http://www1.paho.org/Spanish/AD/DPC/CD/tb-labs-baciloscopia.pdf 
13. Organización Panamericana de la Salud. Manual para el diagnóstico de tuberculosis. Parte 2. Cultivo. Fecha de consulta: 18 de junio de 2016. Disponible en:

http://www1.paho.org/Spanish/AD/DPC/CD/tb-labs-cultivo.pdf

14. Ministerio de Salud y Protección Social. Circular 058 de 2009. Fecha de consulta: 13 de junio de 2016. Disponible en: https://www.minsalud.gov.co/sites/rid/Lists/BibliotecaDigital/ RIDE/DE/DIJ/circular-058-2009.pdf

15. Ministerio de Salud y Protección Social. Resolución 5975 de 2016. Fecha de consulta: 13 de junio de 2016. Disponible en: http://www.sispro.gov.co/recursosapp/SiteAssets/ Resolucion-5975-de-2016-CUPS.pdf

16. Pérez T, Pérez L, Ghaddar N. Caracterización clínico-epidemiológica de la tuberculosis en la edad pediátrica. Santa Clara, Cuba: Universidad de Ciencias Médicas "Dr. Serafín Ruíz de Zárate Ruíz" de Villa Clara; 2013. Fecha de consulta: 8 de marzo de 2016. Disponible en: http://files.sld.cu/enfermeria-pediatria/files/2013/03/mencion-3-caracterizacion-clinicoepidemiologica-de-la-tuberculosis-en-la-edad-pediatrica-santa-clara-2009-2011.pdf

17. Dizdar S, Dzinovic A, Gojak R, Bakalovic G, Selimovic A. Descriptive review of the patients suffering from tuberculosis in the past 10 years treated at Pediatric Clinic of Clinical Center of Sarajevo University. Med Arch. 2014;68:317-8. https://doi.org/10.5455/medarh.2014.68.317-318

18. Haq S, Hussain M, Hrishin J, Abbasi S. Risk factors of tuberculosis in children. Ann Pak Inst Med Sci. 2010;6:50-4.

19. Arteaga A, Vélez E, Salazar O, Morales O, Cornejo J, Valencia D. Características clínicas y sociodemográficas de niños menores de 13 años con diagnóstico confirmado de tuberculosis pulmonar o sin él, en el Hospital Universitario San Vicente de Paúl, Medellín, Colombia, 2007-2008. latreia. 2010;23:227-39.

20. Morcillo N, Imperiale B, Corral J. Tuberculosis pediátrica, MDR-TB y XDR-TB en la provincia de Buenos Aires durante el período 2002-2007. Rev Am Med Resp. 2009;9:5-13.

21. Farga V, Caminero JA. Tuberculosis. Tercera edición. Santiago y Buenos Aires: Editorial Mediterráneo; 2010. p. 409.

22. Arenas N, García A, Coronado S, Beltrán C, Acosta S, Gómez J, et al. Prevalencia de tuberculosis infantil en Armenia, Colombia. Rev Salud Pública. 2010;12:1000-9.

23. Garzón M, Angée D, Llerena C, Orjuela D, Victoria J. Vigilancia de la resistencia de Mycobacterium tuberculosis a los fármacos antituberculosos, Colombia, 2004-2005. Biomédica. 2008;28:319-26. https://doi.org/10.7705/biomedica.v28i3.71

24. Yuen CM, Jenkins HE, Rodríguez CA, Keshavjee S, Becerra MC. Global and regional burden of isoniazid-resistant tuberculosis. Pediatrics. 2015;136e50-9.

https://doi.org/10.1542/peds.2015-0172 